\title{
Method for Determination of Support Length of Daubechies Basis Function for Wavelet MRA based Moving Characteristic Estimation
}

\author{
Kohei Arai \\ Faculty of Science and Engineering \\ Saga University, Saga City \\ Japan
}

\begin{abstract}
Method for determination of support length of Daubechies basis function for wavelet Multi Resolution Analysis: MRA based moving characteristic estimation is proposed. The method based on root mean square difference between original and reconstructed image from just a low frequency component from MRA. Also, one of applications of the method for detection and tracking of moving targets, typhoon and boundary between warm and cold current observed from satellite remote sensing images is shown. It is found that the proposed method allows to detect and to track for moving targets of a typhoon and a boundary in the time series of GOES images.
\end{abstract}

Keywords-Multi-dimensional wavelet transformation; multi resolution analysis: MRA; moving target detection; support length; typhoon movement; boundary detection and tracking

\section{INTRODUCTION}

Moving characteristics such as velocity and vibration of moving objects are being analyzed by taking advantage of the characteristics of time-frequency analysis by wavelet analysis [1]. Especially, the estimation of the moving vector of the moving object with the multi-resolution analysis based on the Daubechies basis function with the finite length support (compact support), which is widely used for the detection of the moving object because of its short support length Has been proposed [2].

A wavelet is a spatial frequency filter, and although it is desirable that its support has a finite length, in many cases the support length is infinite [3]. The Daubechies basis function is a finite length support, and its support length is short, so it is applied to moving object detection. However, there is no report that mentions how to select the optimum support length suitable for the moving speed.

From the viewpoint of making the complexity of the model that expresses the phenomenon appropriate, studies have been conducted on the optimization of base functions based on the information theory evaluation criterion Minimum Description Length: MDL [4].

It is designed to select the basis function that minimizes the code length for data representation. After that, there was a proposal for optimization based on Akaike's Information Criteria: AIC [5]. This optimization method deals with the entire basis function and is suitable for constructing filters such as noise removal. This paper proposes a method to select the optimum support length for the moving speed of a moving object. The validity of the proposed method has been confirmed by taking typhoons and tides with different moving speeds estimated from time-series data of geostationary meteorological satellite images as examples.

Methods related to wavelet analysis, in particular, detection of moving objects [6] and estimation of movement parameters [7] based on continuous and discrete wavelet transforms and multiresolution analysis [8] have been proposed [9,10]. Also proposed are a method that considers tracking of a moving object using a Kalman filter [11] and a method that considers the selection of wavelet parameters that match the motion of the analysis target [12]. Method for support length determination of base function of wavelet for edge and line detection as well as moving object and change detections is proposed [12].

On the other hand, method for car in dangerous action detection by means of wavelet Multi-Resolution Analysis based on appropriate support length of base function is proposed [13]. Meanwhile, prediction method of El Nino Southern Oscillation event by means of wavelet-based data compression with appropriate support length of base function is proposed [14]. Bright band height assignment with precipitation radar data based on Multi-Resolution Analysis: MRA of wavelet analysis is also proposed [15].

Applying a basis function to multidimensional time series data and transforming it into the time-frequency domain is called Discrete Wavelet Transformation (DWT), and its inverse is called Inverse Discrete Wavelet Transformation (IDWT). A method for estimating the movement vector of a moving object from time-series image information using the Daubechies basis function (complete orthonormal system) that can be completely restored by these is proposed. At this time, it is considered that the method for determining the support length of the basis function has not been determined.

This depends on the frame rate of the moving image to be acquired and the moving characteristics (change rate) of the moving object. Although the optimal support length can be determined if the movement characteristics are known, it is customary that the movement characteristics to be obtained are not given in advance. Therefore, a method for determining the optimum support length by some method is required. 
Here, the author proposes an experimental method for determining the optimal support length. That is, some support length candidates that are considered to be appropriate are selected (for example, 2, 4, 8, 16 etc.), DWT based on the Daubechies basis function of those support lengths is applied to only one stage, and then low frequency is applied. Reconstruction (IDWT) is performed using only the components, and the root mean square error (RMSE) of the reconstructed data and the original data is evaluated, and this is the method that maximizes the maximum support length.

Since the energy (power spectrum) of the analysis target data is invariant, when this RMSE is large, it means that the high frequency components could be extracted efficiently. Therefore, it means that the change (movement) component was effectively extracted by DWT / IDWT. The author shows the validity of this proposed method by introducing some examples.

As an application example of the proposed method, a relatively steeply moving typhoon and a relatively slowly changing tide are taken from Geostationary Operational Environmental Satellite (GOES) visible band images acquired on the hourly basis. Determine the optimal support length of Daubechies basis functions for moving analysis of.

The DWT based on this basis function is applied to the original image to separate the original image into lowfrequency and high-frequency components, the low-frequency components are removed and reconstructed to extract edges, and the extracted edge time-series data is obtained.

The method of estimating the movement vector is tried based on this. As a result, it was confirmed that the desired edge could be extracted by DWT and IDWT based on the support length basis function determined based on the proposed method, and it was confirmed that it was effective for the detection and movement tracking of typhoons and tides.

Section 2 describes related research works. Section 3 details the method of constructing basis functions and the definition of their support length. Section 4 proposes the proposed method for analyzing the moving characteristics of moving objects, and Section 5 introduces the above-mentioned case to show that the optimum support length exists and how to obtain it. Finally, the conclusion is stated.

\section{RELATED RESEARCH WORKS}

As for wavelet analysis related research works, bright band height assignment with precipitation radar data based on MultiResolution Analysis: MRA of wavelet analysis is conducted [16]. Also, improvement of secret image invisibility in circulation image with Dyadic wavelet based data hiding with run-length coding is achieved [17].

Extraction of line features from multifidus muscle of CT scanned images with morphological filter together with wavelet multi resolution analysis is made [18]. On the other hand, $\mathrm{CO}_{2}$ concentration change detection in time and space domains by means of wavelet analysis of MRA: Multi Resolution Analysis is conducted [19].
Polarimetric SAR image classification with high frequency component derived from wavelet multi resolution analysis: MRA is proposed [20]. Meanwhile, embedded object detection with radar echo data by means of wavelet analysis of MRA: Multi Resolution Analysis is conducted [21].

Maximum entropy method based blind separation by means of neural network with feature enhancements by wavelet analysis is proposed [22]. On the other hand, method for support length determination of base function of wavelet for edge and line detection as well as moving object and change detections is proposed [23].

Human gait gender classification using 2D discrete wavelet transforms energy is conducted [24]. Meanwhile, visualization of 3D object shape complexity with wavelet descriptor and its application to image retrievals is attempted together with visualization of 3D object shape complexity with wavelet descriptor and its application to image retrievals [25].

Wavelet based image retrieval method is proposed [26]. Meanwhile, DP matching based image retrieval method with wavelet Multi Resolution Analysis: MRA which is robust against magnification of image size is conducted [27]. On the other hand, wavelet based change detection for four dimensional assimilation data in space and time domains is attempted [28].

Method for image source separation by means of independent component analysis: ICA, Maximum Entropy Method: MEM, and wavelet based method is attempted [29]. On the other hand, identification of ornamental plant functioned as medicinal plant based on redundant discrete wavelet transformation is conducted [30].

Method for object motion characteristics estimation based on wavelet Multi-Resolution Analysis: MRA is proposed [31]. Also, identification of ornamental plant functioned as medicinal plant based on redundant discrete wavelet transformation is attempted [32].

Comparative study on discrimination methods for identifying dangerous red tide species based on wavelet utilized classification methods is conducted [33]. On the other hand, identification of ornamental plant functioned as medicinal plant based on redundant discrete wavelet transformation is conducted [34].

Method for car in dangerous action detection by means of wavelet Multi-Resolution Analysis based on appropriate support length of base function is proposed [35]. Meanwhile, improvement of automated detection method for clustered micro calcification base on wavelet transformation and support vector machine is conducted [36].

Prediction method of El Nino Southern Oscillation: ENSO event by means of wavelet based data compression with appropriate support length of base function is proposed [37]. Meanwhile, method for data hiding based on Legall $5 / 2$ (Cohen-Daubechies-Feauveau: CDF 5/3) wavelet with data compression and random scanning of secret imagery data is proposed [38]. 
Image retrieval and classification methods based on Euclidian distance between normalized features including wavelet descriptor are proposed [39]. On the other hand, gender classification method based on gait energy motion derived from silhouettes through wavelet analysis of human gait moving pictures is proposed [40].

Multifidus muscle volume estimation based on three dimensional wavelet Multi Resolution Analysis: MRA with buttocks Computer Tomography: CT images is conducted [41]. In a meantime, method for object motion characteristics estimation based on wavelet Multi resolution Analysis: MRA is also proposed [42].

Gender classification method based on gait energy motion derived from silhouette through wavelet analysis of human gait moving pictures is proposed [43]. On the other hand, method for object motion characteristics estimation based on wavelet Multi resolution Analysis: MRA is proposed [44].

Comparative study of feature extraction components for several wavelet transformations for ornamental plants is conducted [45]. Meanwhile, human gait gender classification using $3 \mathrm{D}$ discrete wavelet transformation feature extraction is attempted [46].

Image retrieval method utilizing texture information derived from Discrete Wavelet Transformation: DWT together with color information is proposed [47]. Meanwhile, noble method for data hiding using steganography Discrete Wavelet Transformation: DWT and cryptography triple Data Encryption Standard: DES is proposed [48].

Phytoplankton discrimination method with wavelet descriptor based shape feature extraction from microscopic images is proposed [49]. Also, change detection method with multi-temporal satellite images based on wavelet decomposition and tiling is proposed [50]. On the other hand, circle feature extraction from remote sensing satellite images based on polar coordinate representation of wavelets is conducted [51].

\section{DISCRETE WAVELET TRANSFORMATION WITH BI- ORTHOGONAL DAUBECHIES BASIS FUNCTION}

\section{A. Discrete Wavelet Transformation}

The Discrete Wavelet Transformation: DWT of a given discrete scalar signal $\eta=\left(\eta_{1}, \eta_{2}, \ldots, \eta_{\mathrm{n}}\right)^{T}$ is described as $C_{n} \eta$ by a square matrix $C_{n}$ composed of a sequence $\left\{p_{k}\right\}$ and a sequence $\left\{q_{k}\right\}$. pi is for low-frequency components, coefficient $q i$ is for high-frequency components, $C_{n}$ divides $f$ into lowfrequency components and high-frequency components.

The given scalar time series is divided into low frequency components and high frequency components by DWT. At this time, for example, the DWT for eight elements having a support length of 2 becomes Eq. (1), and when the support length is 4, it becomes Eq. (2).
$C_{8}^{[2]}\left[\begin{array}{c}\eta_{1} \\ \eta_{2} \\ \eta_{3} \\ \eta_{4} \\ \eta_{5} \\ \eta_{6} \\ \eta_{7} \\ \eta_{8}\end{array}\right]=\left[\begin{array}{ccccc}p_{0} & p_{1} & & & \\ q_{0} & q_{1} & \ldots & & \\ & \vdots & \ddots & & \vdots \\ & & \ldots & p_{0} & p_{1} \\ & & & q_{0} & q_{1}\end{array}\right]\left[\begin{array}{c}\eta_{1} \\ \eta_{2} \\ \eta_{3} \\ \eta_{4} \\ \eta_{5} \\ \eta_{6} \\ \eta_{7} \\ \eta_{8}\end{array}\right]=\left[\begin{array}{c}p_{0} \eta_{1}+p_{1} \eta_{2} \\ q_{0} \eta_{1}+q_{1} \eta_{2} \\ p_{0} \eta_{3}+p_{1} \eta_{4} \\ q_{0} \eta_{3}+q_{1} \eta_{4} \\ p_{0} \eta_{5}+q_{1} \eta_{6} \\ q_{0} \eta_{5}+p_{1} \eta_{6} \\ p_{0} \eta_{7}+p_{1} \eta_{8} \\ q_{0} \eta_{7}+q_{1} \eta_{8}\end{array}\right]$

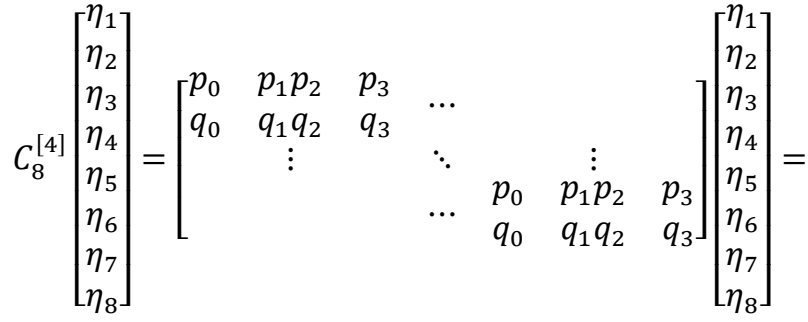

$\left[\begin{array}{l}p_{0} \eta_{1}+p_{1} \eta_{2}+p_{2} \eta_{1}+p_{3} \eta_{2} \\ q_{0} \eta_{1}+q_{1} \eta_{2}+q_{2} \eta_{1}+q_{3} \eta_{2} \\ p_{0} \eta_{3}+p_{1} \eta_{4}+p_{2} \eta_{3}+p_{3} \eta_{4} \\ q_{0} \eta_{3}+q_{1} \eta_{4}+q_{2} \eta_{3}+q_{3} \eta_{4} \\ p_{0} \eta_{5}+q_{1} \eta_{6}+p_{2} \eta_{5}+q_{3} \eta_{6} \\ q_{0} \eta_{5}+p_{1} \eta_{6}+q_{2} \eta_{5}+p_{3} \eta_{6} \\ p_{0} \eta_{7}+p_{1} \eta_{8}+p_{2} \eta_{7}+p_{3} \eta_{8} \\ q_{0} \eta_{7}+q_{1} \eta_{8}+q_{2} \eta_{7}+q_{3} \eta_{8}\end{array}\right]$

The coefficients of base functions with different That is, the support length is different even if the order is the same. This is called a two-dimensional DWT. Similar to the twodimensional DWT, the three-dimensional DWT is realized by performing filter processing on each dimension as shown in Fig. 1. Furthermore, multivariate multidimensional DWT is performed by filtering the dimension of each variable.

\section{B. Support Length}

Daubechies support lengths are obtained as solutions of simultaneous equations as shown in the following,

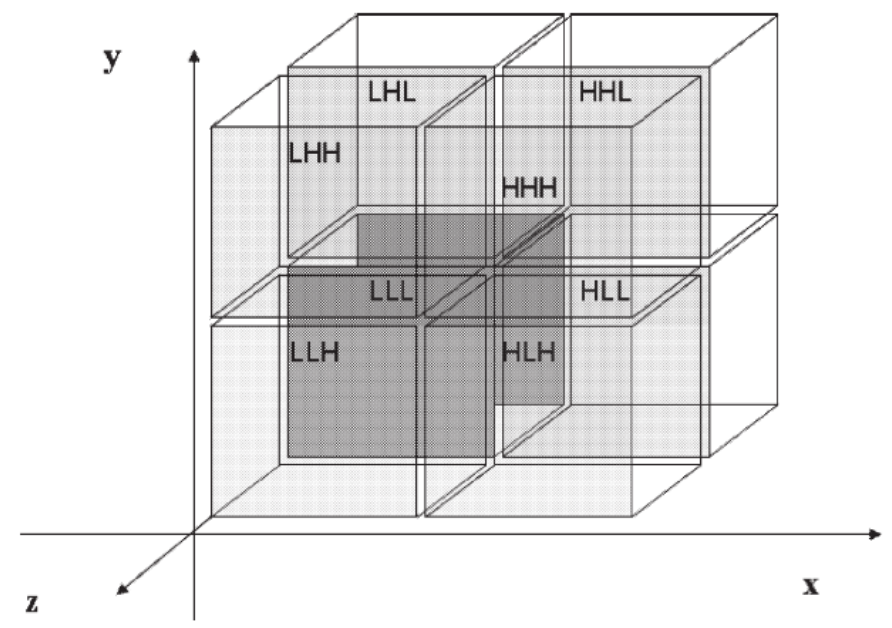

Fig. 1. 3D Discrete Wavelet Transformation (3D DWT). 
When the base function is Daubechies and the support length is 2, the coefficient is obtained by solving the simultaneous equations of Eq. (3). When the support length is 4, the coefficient is obtained so as to satisfy Eq. (4). Further, in the case of an arbitrary support length (Sup), the coefficient of the basis function can be obtained by solving Eq. (5) simultaneously.

$$
\begin{aligned}
& C_{n}^{[2]^{T}} C_{n}^{[2]}=I_{n} \\
& p_{0}+p_{1}=\sqrt{2}, \\
& q_{0}=p_{1}, q_{1}=-p_{1}, 0^{0} q_{0}+1^{0} q_{1}=0 \\
& C_{n}^{[4]^{T}} C_{n}^{[4]}=I_{n} \\
& p_{0}+p_{1}+p_{2}+p_{3}=\sqrt{2}, \\
& q_{0}=p_{3}, q_{1}=-p_{2}, q_{2}=p_{1}, q_{3}=-p_{0}, \\
& 0^{0} q_{0}+1^{0} q_{1}+2^{0} q_{2}+3^{0} q_{3}=0 \\
& 0^{1} q_{0}+1^{1} q_{1}+2^{1} q_{2}+3^{1} q_{3}=0 \\
& C_{n}^{[s u p]^{T}} C_{n}^{[s u p]}=I_{n} \\
& \text { sup-1 } \\
& \sum_{j=0} p_{j}=\sqrt{2}, \\
& q_{j}=(-1)^{j} p_{((s u p-1)-j}, j=0,1,2, \ldots,(\text { sup }-1) \\
& \sum_{j=0}^{\text {sup-1 }} j^{r} q_{j}=0, r=0,1,2, \ldots,\left(\frac{\text { sup }}{2}-1\right)
\end{aligned}
$$

This is called a two-dimensional wavelet transform. The three-dimensional wavelet transform is realized by performing a filtering process on each dimension, like the two-dimensional wavelet transform. Furthermore, the multivariate multidimensional wavelet transform is performed by filtering the dimension of each variable.

\section{Multi Dimensional Discrete Wavelet Transformation}

By applying the DWT based on the basis function obtained by the above method to the two-dimensional image data, it is possible to obtain an arbitrary frequency component included in the image. In addition, in the case of time-series image data of moving images, it is possible to investigate any frequency component at any time by applying 3D DWT.

Detection of a moving object included in moving image data, tracking, and analysis of movement characteristics are performed by the following procedure. First, the 2D image of each frame number of the moving image is filtered in the horizontal direction of the target 2D image, and divided into two regions of low frequency component (L component) and high frequency component (H component).

Next, filter processing is performed in the vertical direction of each of these two areas. By the above processing, the twodimensional discrete signal is divided into four (LL component, LH component, HL component, HH component). This is the two-dimensional DWT. Here, if $f$ is the one-dimensional scalar time-series data and $F$ is the spectrum data after DWT, DWT is expressed by Eq. (6).
$\mathrm{F}=\mathrm{Cnf}$

Since the discrete wavelet transform based on Daubechies basis function is considered,

$\mathrm{CnCn}^{\mathrm{T}}=\mathrm{I}$

Therefore, the original time series data can be completely restored by reconstructing after division (reverse transformation after transformation). Also, the 3D DWT for $f_{x, y, z}$ of $3 \mathrm{D}$ data is as follows,

$F=\left[\operatorname{Cn}\left[\operatorname{Cm}\left[\mathrm{Cl} f_{x, y, z}\right]^{\mathrm{T}}\right]^{\mathrm{T}}\right]^{\mathrm{T}}$

The change can be extracted by applying IDWT only to the high frequency components after DWT. For example, if DWT is applied to a two-dimensional image and IDWT is performed with the elements of the LL and LH or LL and HL components set to 0 , the two-dimensional reconstructed image extracts vertical or horizontal change (moving) components. It has been done.

The three-dimensional reconstructed image is also obtained by applying the three-dimensional DWT of Fig. 1 to the threedimensional moving image data for the change component in the time axis direction and performing IDWT with the LLL and LHL or LLL and HLL component elements set to 0 . Means that the time change of the vertical or horizontal change (movement) component is extracted.

\section{Moving Characteristic Estimation}

The moving direction and moving speed are obtained through the above process. At this time, the DWT based on the Daubechies basis function can accurately extract the spatiotemporal changes of the moving object. At that time, it is necessary to appropriately select the basis function to be used and its support length. The basis function may be a perfect orthonormal system, but the support length depends on the movement characteristics to be extracted and the frame rate in moving image acquisition, so the best choice is desired.

\section{E. Method for Optimum Support Length Determination}

The Daubechies basis function is selected because it is necessary to be able to completely restore by wavelet transformation and inverse transformation for the movement tracking analysis of the object. The optimum support length differs depending on the movement characteristics of the object and the image acquisition conditions. If the low frequency component is extracted by applying DWT to the multidimensional time series data with time axis and the Root Mean Square Error: RMSE between it and the original image is small, it can be said that the edge component (high frequency component) included in the original image is small. If this difference is large, it means that the edge was extracted most efficiently.

Therefore, in order to determine the optimal support length for edge extraction, DWTs with different support lengths are applied one stage, and the RMSEs of the resulting lowfrequency component image and the original image are examined, and this is the optimal support length with a large support length. Propose a method of lengthening. Attention should be paid to the effect of noise enhancement associated with edge extraction. 
Wavelet analysis has the function of a filter bank, and it is possible to select the optimum part of the filter bank by adjusting the support length. It is necessary to optimize the support length to prevent the noise from being overemphasized.

This support length depends on the moving speed of the object and the observation speed, and if the observation conditions are the same, there is a support length suitable for the moving speed of the object. That is, qualitatively, it can be said that a basis function with a long support length is suitable for analyzing a phenomenon in which an object moves fast, and conversely, a short support length is suitable for an object moving slowly. Since temporal and spatial changes are generally irrelevant, the author first proposes a method for determining the optimal support length in the image space, and then determining the optimal support length on the time axis.

\section{IV.EXPERIMENT}

Here, typhoon and tide detection in GOES images and movement tracking analysis are illustrated as an example of change characteristic analysis by DWT based on Daubechies basis function for change phenomenon of time series data of 2D image.

\section{A. GOES Data used in the Experiment}

The author selected the following typical typhoons with fast moving speeds and typical tides off Sanriku with relatively slow moving speeds, and obtained the support lengths of the optimum basis functions for each moving speed.

1) Typhoon: IR1 $(10.2-11.2 \mu \mathrm{m})$ data of the meteorological satellite GOES-9 acquired on an hourly basis from October 17, 2004, 19:00 (JST) to October 19, 02:00 (JST).

2) Tide: IR1 $(10.2-11.2 \mu \mathrm{m})$ data of the meteorological satellite GOES-9 acquired on an hourly basis from 00:00 (JST) on October 13, 2008 to 07:00 (JST) on October 14th, 2008.

These images have an image size of 640 x 480 pixels and an image file format PGM (Portable Gray Map). From the data, the area around the typhoon and off Sanriku was extracted as an image size of $256 \times 265$ pixels and used for the experiment. Some GOES images of typhoons and tides are shown in Fig. 2(a) and (b), respectively.

\section{B. Optimal Support Length Determination Method for Edge Extraction based on RMSE between Reconstructed Image and Original Image by DWT / IDWT}

DWT based on Daubechies basis functions with support lengths of 2, 4, 8 and 16 was applied, IDWT was performed using only the LL component, and the RMSE with the original image was evaluated. The reconstructed image and RMSE are shown in Fig. 3 (a) and (b). In the figure, each data indicates the RMSE in each frame.
In the case of the typhoon (a), the RMSE is large up to the first 3 frames and from the last 3 frames, and it can be seen that the time change is sharp. It is also clear that the RMSE of the tide of (b) is low overall, the RMSE is large from the beginning up to about 8 frames, and there is a sharp temporal change with the movement of clouds. It can be seen that the time change is extremely low in the subsequent frames, and there is little movement of the tide.

The average and standard deviation of RMSE are shown in Table I(a) and (b). From these, it can be seen that the optimum support length is 8 for each of the typhoons and the tides off Sanriku, since the flatness of the RMSE is the largest.

\section{Verification of the Proposed Method by the Number of Extracted Edge Pixels}

A threshold of 240 DN (maximum pixel value); the extracted edge image obtained by binarization using $255 \mathrm{DN}$ ) is shown in Fig. 4(a) and (b). Table II shows the average and standard deviation of the number of edge pixels extracted by DWT / IDWT with the support length changed to 2, 4, 8 and 16 .

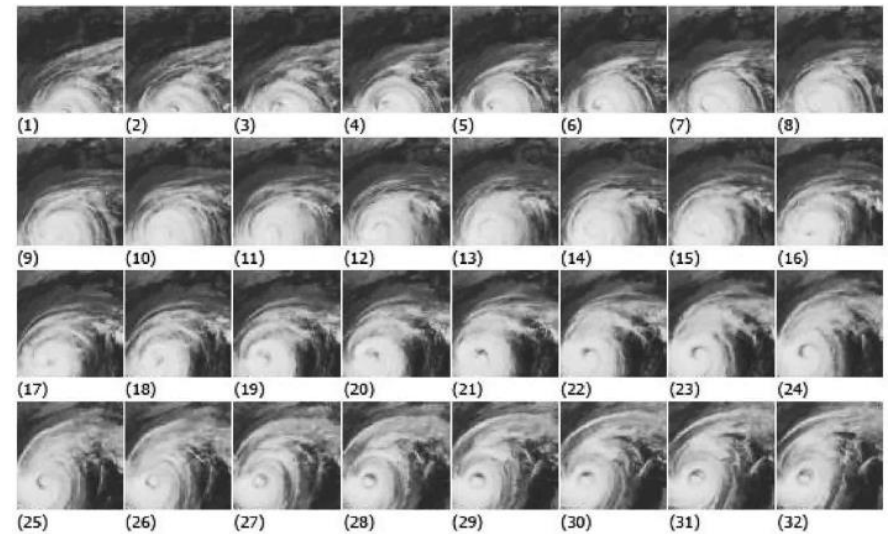

(a) GOES IR-1 imagery data of the typhoon acquired during from 19:00 on October 17 to 2:00 on October 19 in 2004

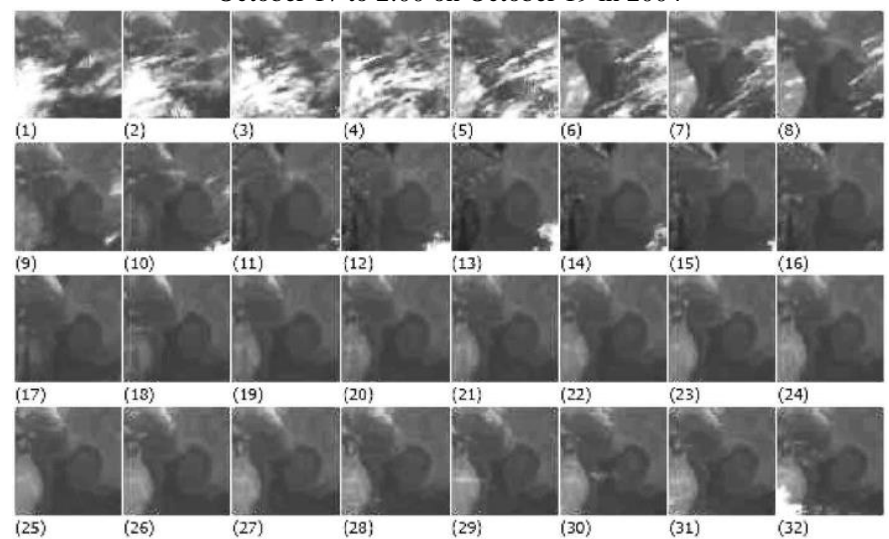

(b) GOES IR-1 imagery data of current rip in Sanriku offshore acquired during from 0: 00 on October 13 to 7: 00 on October 14 in 2004

Fig. 2. The Time Series of Original GOES Imagery Data (32 Frames) of Japanese Vicinity. 

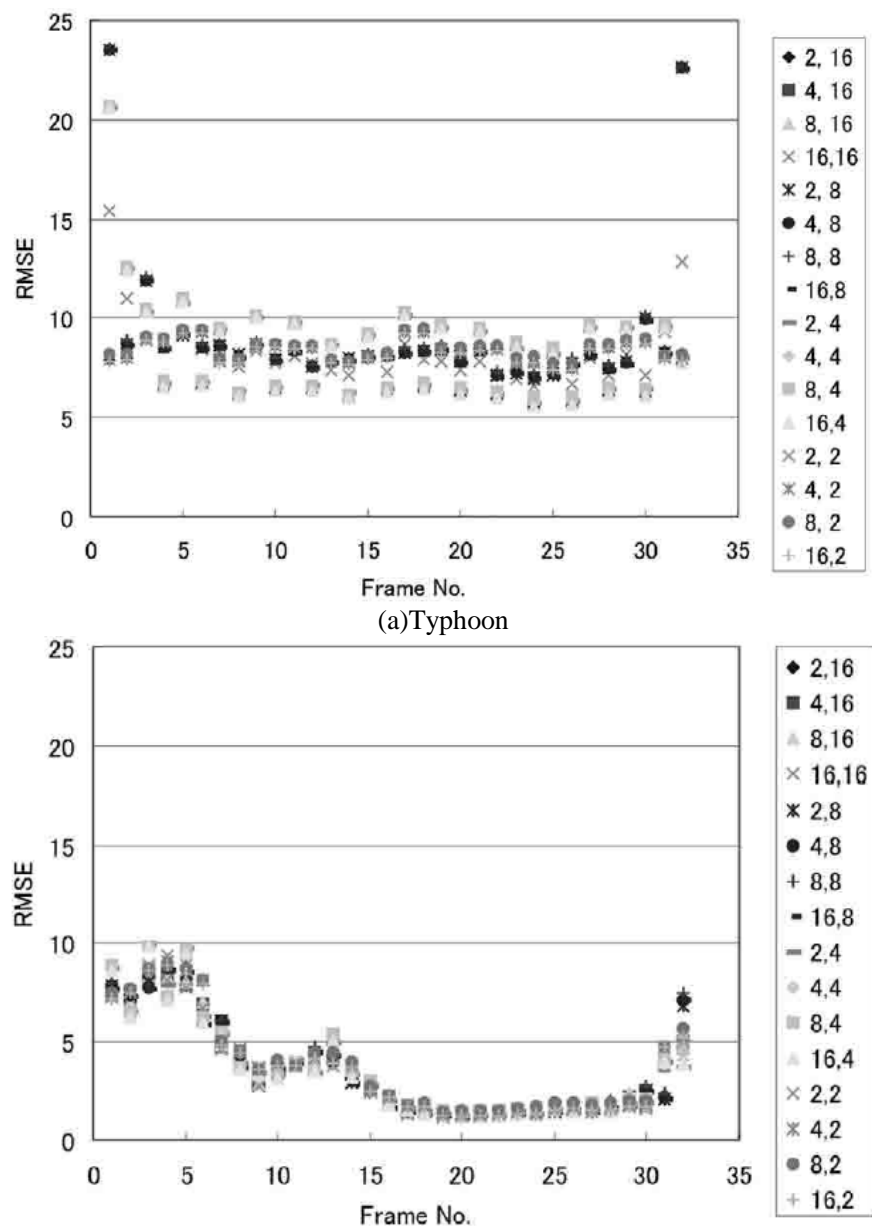

(b) Current rip in Sanriku offshore.

Fig. 3. RMSE between the Original and Reconstructed Images without LL Component by using Discrete Wavelet Transformation based on Daubechies Mother Wavelet Function with the different Support Length.

TABLE I. AVE.: AVERAgE AND STD.: StANDARD DEVIATION OF RMSE: ROOT MEAN SQUARE ERROR BETWEEN THE ORIGINAL IMAGE AND RECONSTRUCTED IMAGE WITHOUT LL COMPONENT FROM THE TRANSFORMED IMAGE WITH DISCRETE WAVELET TRANSFORMATION BASED ON DAUBECHIES MOTHER WAVELET FUNCTION WITH SUPPORT LENGTH OF 2 , 4,8 AND 16

(A) TYPHOON

\begin{tabular}{|l|l|l|l|r|l|}
\hline SupportL. & 2 & 4 & 8 & 16 & \\
\hline Ave. & RMSE & 8.612 & 8.629 & 8.950 & 8.513 \\
\hline Std. & RMSE & 2.376 & 2.759 & 2.989 & 2.547 \\
\hline
\end{tabular}

(B) CURRENT RIP IN SANRIKU OFFSHORE

\begin{tabular}{|l|l|l|l|l|l|}
\hline SupportL. & 2 & 4 & 8 & 16 & \\
\hline Ave. & RMSE & 3.574 & 3.594 & 3.741 & 3.487 \\
\hline Std. & RMSE & 2.525 & 2.443 & 2.411 & 2.444 \\
\hline
\end{tabular}

From Table II(a), the optimum support length for edge extraction and movement analysis for the tide off Sanriku is considered to be 2 (when the support length is 2 , the number of flat edge pixels is $0.5 \%$ compared to when the support length is 8 .

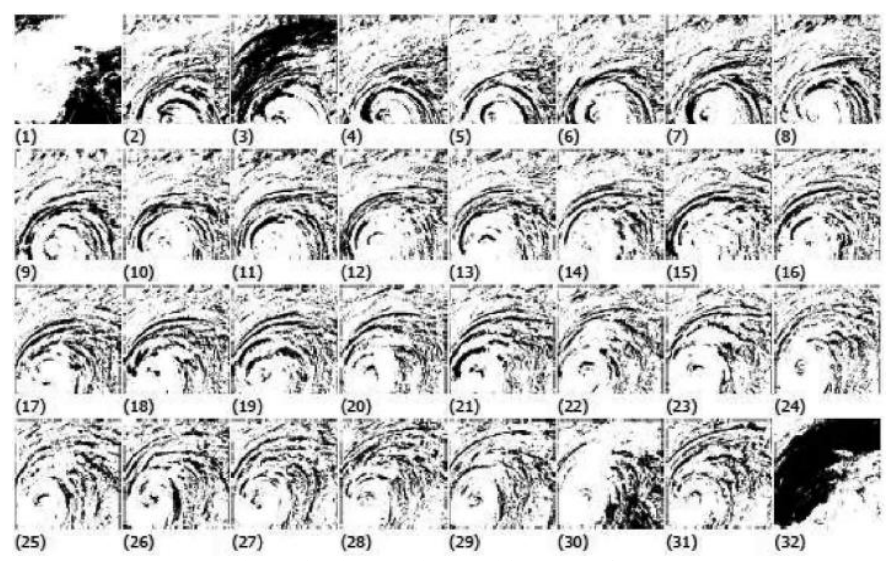

(a) Typhoon in the southern China sea of ocean area

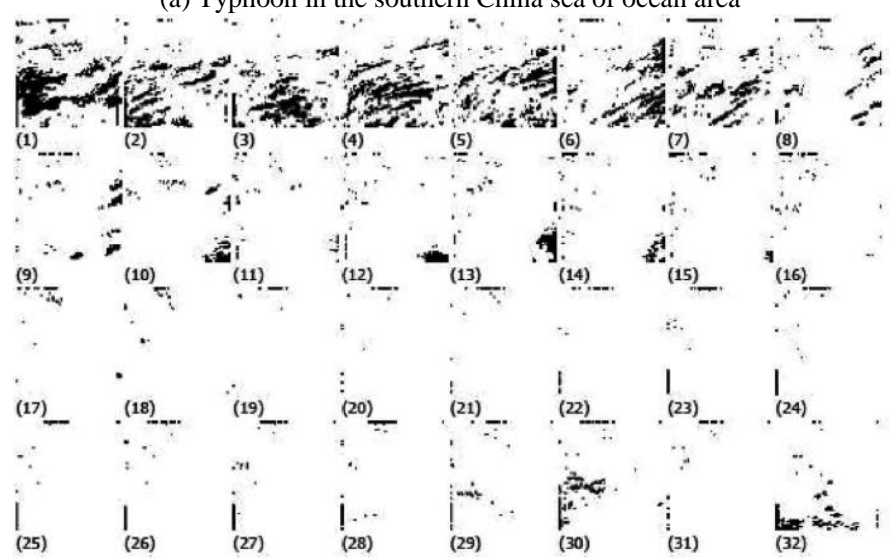

(b) Current rip in Sanriku offshore

Fig. 4. Example of Detected Edge Images of(a) Typhoon and (b) Current Rip in Sanriku offshore Obtained from Binarized Images with the Threshold of 240 Digital Number: DN (255 in Maximum DN)of Reconstructed Images without LL Component after the Discrete Wavelet Transformation with Daubechies Mother Wavelet Function with Support Length of 8

TABLE II. AVE: AVERAGE AND STD.: STANDARD DEVIATION OF RMSE: ROOT MEAN SQUARE ERROR BETWEEN THE ORIGINAL IMAGE AND RECONSTRUCTED IMAGE WITHOUT LLL COMPONENT FROM THE

TRANSFORMED IMAGE WITH DISCRETE WAVELET TRANSFORMATION BASED ON DAUBECHIES MOTHER WAVELET FUNCTION WITH SUPPORT LENGTH OF 2 , 4, 8 AND 16

\begin{tabular}{|l|l|l|l|l|l|}
\hline Support Length. & 2 & 4 & 8 & 16 & \\
\hline Ave. & RMSE & 14.292 & 13.229 & 10.860 & 20.474 \\
\hline Std. & RMSE & 8.671 & 7.713 & 6.747 & 30.109 \\
\hline
\end{tabular}

This is because, as can be seen from the standard deviation, the edges accompanying the movement of clouds included in the images of frame numbers 1-5 are large and the standard deviation is raised. Therefore, taking this into consideration, it was confirmed that the DWT / IDWT based on basis functions with a support length of 8 is optimal for spatiotemporal edge extraction. In addition, the effect of noise increase due to edge enhancement can be seen from Fig. 4(a), (b) and Table II. When the edge component is large like a typhoon image, the influence is large. The standard deviation in the table reflects the noise component, but when the support length is optimum, the noise component, that is, the standard deviation, is small. 


\section{Optimal Support Length for Moving Vector Detection along the Time Axis}

As mentioned above, temporal changes and spatial changes are originally different. Therefore, it is better to determine the optimal support length in the time axis direction separately from spatial edge extraction. The method is as follows:

1) Determine the optimal support length for extracting the spatial change based on the RMSE described above by twodimensional DWT / IDWT.

2) Next, find the optimum support length for detecting the movement vector in the time axis direction. Therefore, first, a two-dimensional DWT based on the optimal support length basis function found in (1) is applied to the original image to generate a time-series DWT transformed image data set.

3) After that, one-dimensional DWT is applied to the time-series data in which the transformed image is arranged in the time axis direction for each pixel, and IDWT is performed using only the L component.

4) After that, the RMSE between the reconstructed image and the original image is evaluated to determine the optimal support length for extracting changes in the time axis direction. At this time, the changes in the RMSE time axis direction for each support length were compared with the RMSE time average.

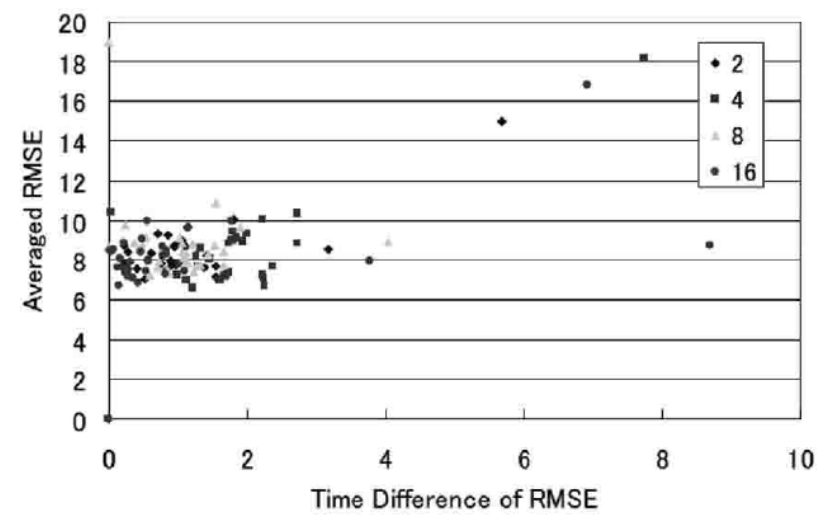

(a) Typhoon.

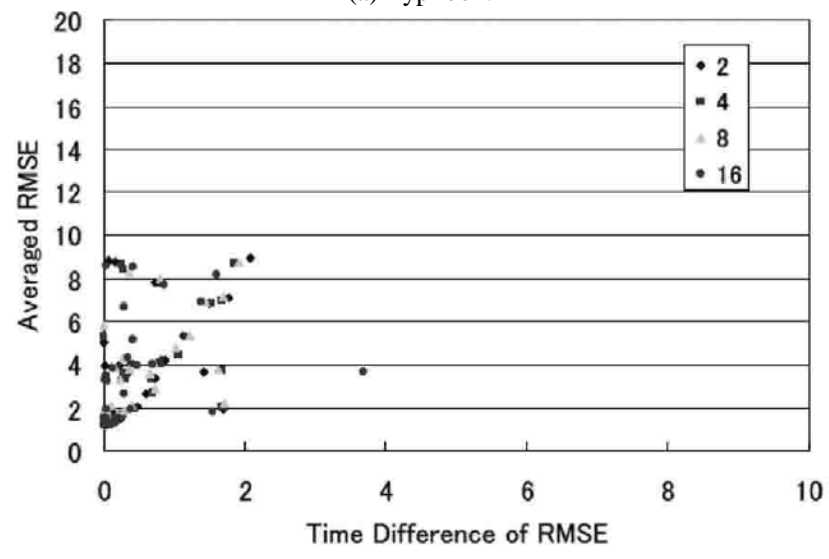

(b) Current Rip.

Fig. 5. Most Appropriate Support Length of Daubechies Mother Wavelet Function for Detection of Moving Targets from Time Series of GOES Imagery Data.
As shown in Fig. 5, it can be seen that, as a general tendency, the time flatness of RMSE is large when the time change is large. In addition, it can be seen that the optimum support length is different when the moving object has a steep temporal change such as a typhoon and when it moves slowly like a tide. In other words, the temporal change of RMSE in the case of the tide is concentrated within 2 and the time average of RMSE is also within 9.

A larger time change than that is the steep part of the time change associated with the movement of clouds in frame numbers 1-5. On the other hand, in the case of a typhoon, the time variation of RMSE is concentrated within about 4 and the time average of RMSE reaches 18. Furthermore, the support length of the part of the RMSE that has a large change over time is often 16 and is followed by 8,4 , and 2 .

Therefore, it can be seen that the DWT / IDWT analysis based on the basis function with a long support length is suitable for detecting and tracking moving objects with large temporal changes. In summary, it can be construed that when detecting and tracking a phenomenon that changes rapidly, it is necessary to extend the support length and view the observation data over a wide time range.

\section{CONCLUSION}

In this paper, the author first show that spatiotemporal feature extraction based on Daubechies basis functions is effective for detecting moving objects such as typhoons, clouds, and tidal waves in Earth observation satellite image data, and analyzing movement vectors.

The author proposed a method for optimizing the support length of the basis function used in that case. In other words, the three-dimensional discrete wavelet transform based on the basis function of the support length in the assumed range $(1,2$, $4,8,16,32$, etc.) is applied to the time-series earth observation satellite image data in one stage, and low frequency components (LLL Component (reverse discrete wavelet transform) is used to evaluate the squared deviation between the original data and the reconstructed data, and this is large (edge component due to movement of object: high-frequency component is extracted greatly). The optimum support length is determined with such support length.

The author evaluated the effectiveness of the proposed method by applying it to time-series data of geostationary meteorological satellite GOES images, and found that the Daubechies basis function, which has a long support length, can be used to analyze the motion vector of an object that moves rapidly like a typhoon. It was confirmed that the spatiotemporal feature extraction based on the spatiotemporal feature extraction based on the Daubechies basis function, which has a short support length, is effective for the motion vector analysis of the phenomenon such as the tidal movement.

\section{VI.FUTURE RESEARCH WORKS}

In the future, the author will continue to validate of the proposed method with a variety of time series of data. 


\section{ACKNOWLEDGMENT}

This is a report of the research results (or part of it) by the Grant-in-Aid for Scientific Research (Special Researcher Incentive). The author would like to express my gratitude to the Grant provider. The author would like to thank Dr. Kaname Seto of former student of Saga University and Dr. Leland M. Jameson of Naval Research Laboratory for their contribution of this study. The author, also, would like to thank Professor Dr. Hiroshi Okumura and Professor Dr. Osamu Fukuda for their valuable discussions.

\section{REFERENCES}

[1] Kohei Arai, Basic Theory of Wavelet Analysis, Morikita Publishing (November 2000).

[2] Kohei Arai, Leland Jameson, How to use earth observation satellite data by wavelet analysis, Morikita Publishing (July 2001).

[3] Kohei Arai, Self-study wavelet analysis, published by Modern Science Co., Ltd. (June 2006).

[4] Saito, N., Simultaneous noise suppression and signal compression using a library of orthogonal bases and the minimum description length criterion, In Wavelets in Geophysics, Foufoula-Georgiou, E.and Kumar,P.(edt),Academic Press,pp.299-324, 1994.

[5] Yuki Matsushima, Shingo Shirahata, Wataru Sakamoto, Selection of Wavelet Basis Functions by AIC, Applied Statistics, 33, 2, 201-219, 2004.

[6] Corbett J., Leduc J.-P., and Kong M.,Analysis of de-formational transformations with spatiotemporal continuous wavelet transforms, in Proceedings of IEEE-ICASSP, 1999.

[7] Duval-Destin M . and Murenzi R., Spatio-temporal wavelets Applications to the analysis of moving patterns, Progress in Wavelet Analysis and Applications, Editions Fronti $\square$ eres, Gif-sur-Yvette, France, 1993.

[8] J.-P.Leduc, F.M ujica, R.M urenzi, and M .J.T. Smith, Spatio temporal Wavelets : A Group-The oretic Construction for Motion Estimation and Tracking. SIAM J. Appl. Math. Society for Industrial and Applied Mathematics 61, 2, 596-632, 2000

[9] F.M ujica, J-P.Leduc, R.M urenzi and M.J.T. Smith, A New Motion Parameter Estimation Algorithm Based on the Continuous Wavelet Transform IEEE Transactions on Image Processing, 9, 5, 873-888, 2000.

[10] Bing Wang, Rong Chun Zhao,Motion tracking using the continuous wavelet transform and EKF models, Proceedings of the International Computer Congress 2004, 155-161, World Scientific Publishing Co. Pte. Ltd., 2004.

[11] BraultP., A New Scheme For Object-Oriented Video Compression And Scene Analysis, Based On Motion Tuned Spatio-Temporal Wavelet Family and Trajectory Identification, IEEE-ISSPIT03, 2003.

[12] Kohei Arai, Method for support length determination of base function of wavelet for edge and line detection as well as moving object and change detections, International Journal of Research and Reviews on Computer Science, 2, 4, 1133-1139, 2011.

[13] Kohei Arai, Tomoko Nishikawa, Method for car in dangerous action detection by means of wavelet Multi-Resolution Analysis based on appropriate support length of base function, International Journal of Advanced Research in Artificial Intelligence, 2, 4, 13-17, 2013.

[14] Kohei Arai, Prediction method of El Nino Southern Oscillation event by means of wavelet based data compression with appropriate support length of base function, International Journal of Advanced Research in Artificial Intelligence, 2, 8, 16-20, 2013.

[15] Kohei Arai and M.Saka, Bright band height assignment with precipitation radar data based on Multi-Resolution Analysis: MRA of wavelet analysis, Advances in Space Research, 37, 12, 2197-2201, 2006.

[16] Kohei Arai and Yuji Yamada, Improvement of secret image invisibility in circulation image with Dyadic wavelet based data hiding with runlength coding, International Journal of Advanced Computer Science and Applications, 2, 7, 33-40, 2011.
[17] Kohei Arai, Yuichiro Eguchi and Yoichiro Kitajima, Extraction of line features from multifidus muscle of CT scanned images with morphological filter together with wavelet multi resolution analysis, International Journal of Advanced Computer Science and Applications, 2, 8, 60-66, 2011.

[18] Kohei Arai, $\mathrm{CO}_{2}$ concentration change detection in time and space domains by means of wavelet analysis of MRA: Multi Resolution Analysis, International Journal of Advanced Computer Science and Applications, 2, 8, 82-86, 2011.

[19] Kohei Arai, Polarimetric SAR image classification with high frequency component derived from wavelet multi resolution analysis: MRA, International Journal of Advanced Computer Science and Applications, 2, 9, 37-42, 2011.

[20] Kohei Arai, Embedded object detection with radar echo data by means of wavelet analysis of MRA: Multi Resolution Analysis, International Journal of Advanced Computer Science and Applications, 2, 9, 27-32, 2011.

[21] Kohei Arai, Maximum entropy method based blind separation by means of neural network with feature enhancements by wavelet analysis, International Journal of Research and Reviews on Computer Science, 2, 4, 1116-1122, 2011.

[22] Kohei Arai, Method for support length determination of base function of wavelet for edge and line detection as well as moving object and change detections, International Journal of Research and Reviews on Computer Science, 2, 4, 1133-1139, 2011.

[23] Kohei Arai, Rosa Andrie, Human gait gender classification using 2D discrete wavelet transforms energy, International Journal of Computer Science and Network Security, 11, 12, 62-68, 2011.

[24] Kohei Arai, Visualization of 3D object shape complexity with wavelet descriptor and its application to image retrievals, Journal of Visualization, DOI:10.1007/s, 12650-011-0118-6, 2011.

[25] Kohei Arai, Visualization of 3D object shape complexity with wavelet descriptor and its application to image retrievals, Journal of Visualization, 15, 2, 155-166, 2012.

[26] Kohei Arai, C.Rahmad, Wavelet based image retrieval method, International Journal of Advanced Computer Science and Applications, 3, 4, 6-11, 2012.

[27] Kohei Arai, DP matching based image retrieval method with wavelet Multi Resolution Analysis: MRA which is robust against magnification of image size, International Journal of Research and Review on Computer Science, 3, 4, 1738-1743, 2012.

[28] Kohei Arai, Wavelet based change detection for four dimensional assimilation data in space and time domains, International Journal of Advanced Computer Science and Applications, 3, 11, 71-75, 2012.

[29] Kohei Arai, Method for image source separation by means of independent component analysis: ICA, Maximum entropy method, and wavelet based method, International Journal of Advanced Computer Science and Applications, 3, 11, 76-81, 2012.

[30] Kohei Arai, I.N.Abdulah, H.Okumura, Identification of ornamental plant functioned as medicinal plant based on redundant discrete wavelet transformation, International Journal of Computer Applications, 61, 1, 34-38, 2013.

[31] Kohei Arai Method for object motion characteristics estimation based on wavelet Multi-Resolution Analysis: MRA, International Journal of Advanced Research in Artificial Intelligence, 2, 1, 25-32, (2013).

[32] Kohei Arai, Indra Nugraha Abdulah, Hiroshi Okumura, Identification of ornamental plant functioned as medicinal plant based on redundant discrete wavelet transformation, International Journal of Computer applications, 61, 1, 1-5, (2013).

[33] Kohei Arai, Comparative study on discrimination methods for identifying dangerous red tide species based on wavelet utilized classification methods, International Journal of Advanced Computer Science and Applications, 4, 1, 95-102, 2013.

[34] Kohei Arai, Indra Nugraha Abdullah, Hiroshi Okumura, Identification of ornamental plant functioned as medicinal plant based on redundant discrete wavelet transformation, International Journal of Advanced Research in Artificial Intelligence, 2, 3, 60-64, 2013.

[35] Kohei Arai, Tomoko Nishikawa, Method for car in dangerous action detection by means of wavelet Multi-Resolution Analysis based on 
appropriate support length of base function, International Journal of Advanced Research in Artificial Intelligence, 2, 4, 13-17, 2013.

[36] Kohei Arai, Indra Nugraha Abdullah, Hiroshi Okumura, Rie Kawakami, Improvement of automated detection method for clustered micro calcification base on wavelet transformation and support vector machine, International Journal of Advanced Research in Artificial Intelligence, 2, 4, 23-28, 2013.

[37] Kohei Arai, Prediction method of El Nino Southern Oscillation event by means of wavelet based data compression with appropriate support length of base function, International Journal of Advanced Research in Artificial Intelligence, 2, 8, 16-20, 2013.

[38] Kohei Arai, Method for data hiding based on Legall 5/2 (CohenDaubechies-Feauveau: CDF 5/3) wavelet with data compression and random scanning of secret imagery data, International Journal of Wavelets Multi Solution and Information Processing, 11, 4, 1-18, B60006 World Scientific Publishing Company, DOI: I01142/SO219691313600060, 1360006-1, 2013.

[39] Kohei Arai, Image retrieval and classification method based on Euclidian distance between normalized features including wavelet descriptor, International Journal of Advanced Research in Artificial Intelligence, 2, 10, 19-25, 2013.

[40] Kohei Arai, Rosa Andrie Asmara, Gender classification method based on gait energy motion derived from silhouettes through wavelet analysis of human gait moving pictures, International Journal of Information Technology and Computer Science, 6, 3, 1-11, 2014.

[41] Kohei Arai, Multifidus muscle volume estimation based on three dimensional wavelet Multi Resolution Analysis: MRA with buttocks Computer Tomography: CT images, International Journal of Advanced Research in Artificial Intelligence, 2, 12, 9-15, 2013.

[42] Kohei Arai, Method for object motion characteristics estimation based on wavelet Multi resolution Analysis: MRA, International Journal of information Technology and Computer Science, 6, 1, 41-49, DOI: 10.5815/ijitcs, 2014.01.05, 2014.

[43] Kohei Arai, Rosa Andrie Asmara, Gender classification method based on gait energy motion derived from silhouette through wavelet analysis of human gait moving pictures, International Journal of Information technology and Computer Science, 5, 5, 12-17, 2013.

[44] Kohei Arai, Method for object motion characteristics estimation based on wavelet Multi resolution Analysis: MRA, International Journal of Information Technology and Computer Science, 1, 41-49, DOI: 10.5815/ijitcs, 2014.01.05, 2014.

[45] Kohei Arai, Indra Nugraha Abdullar, H.Okumura, Comparative study of feature extraction components for several wavelet transformations for ornamental plants, International Journal of Advanced Research in Artificial Intelligence, 3, 2, 5-11, 2014.
[46] Kohei Arai, Rosa Andrie Asmara, Human gait gender classification using 3D discrete wavelet transformation feature extraction, International Journal of Advanced Research in Artificial Intelligence, 3, 2, 12-17, 2014.

[47] Kohei Arai, Cahya Rahmad, Image Retrieval Method Utilizing Texture Information Derived from Discrete Wavelet Transformation Together with Color Information, International Journal of Advanced Research on Artificial Intelligence, 5, 10, 1-6, 2016.

[48] Cahya Rahmed Kohei Arai, Arief Prasetyo, Noriza Arigki, Noble Method for Data Hiding using Steganography Discrete Wavelet Transformation and Cryptography Triple Data Encryption Standard: DES, International Journal of Advanced Computer Science and Applications IJACSA, 9, 11, 261-266, 2018.

[49] Kohei Arai, Phytoplankton discrimination method with wavelet descriptor based shape feature extraction from microscopic images, Journal of RIMS Signal and time frequency analysis, Kokyuroku edited by Ryuichi Ashino, Kyoto University, 50-86, 2019.

[50] Kohei Arai, Change Detection Method with Multi-temporal Satellite Images based on Wavelet Decomposition and Tiling, Journal of Advanced Computer Science and Applications, Vol. 12, No. 3, 56-61, 2021.

[51] Kohei Arai, Circle Feature Extraction from Remote Sensing Satellite Images Based on Polar Coordinate Representation of Wavelets, FICC 2021: Advances in Information and Communication, Springer, vol.1, pp 558-566, 2021.

\section{AUTHORS' PROFILE}

Kohei Arai, He received BS, MS and PhD degrees in 1972, 1974 and 1982, respectively. He was with The Institute for Industrial Science and Technology of the University of Tokyo from April 1974 to December 1978 also was with National Space Development Agency of Japan from January, 1979 to March, 1990. During from 1985 to 1987, he was with Canada Centre for Remote Sensing as a Post Doctoral Fellow of National Science and Engineering Research Council of Canada. He moved to Saga University as a Professor in Department of Information Science on April 1990. He was a councilor for the Aeronautics and Space related to the Technology Committee of the Ministry of Science and Technology during from 1998 to 2000 . He was a councilor of Saga University for 2002 and 2003. He also was an executive councilor for the Remote Sensing Society of Japan for 2003 to 2005 . He is a Science Council of Japan Special Member since 2012. He is an Adjunct Professor of University of Arizona, USA since 1998. He also is Vice Chairman of the Science Commission "A" of ICSU/COSPAR since 2008 then he is now award committee member of ICSU/COSPAR. He wrote 55 books and published 620 journal papers as well as 450 conference papers. He received 66 of awards including ICSU/COSPAR Vikram Sarabhai Medal in 2016, and Science award of Ministry of Mister of Education of Japan in 2015. $\mathrm{He}$ is now Editor-in-Chief of IJACSA and IJISA. http://teagis.ip.is.sagau.ac.jp/index.html.. 